\title{
Bimanual simultaneous movements and hemispheric dominance: Timing of events reveals hard-wired circuitry for action, speech, and imagination
}

\author{
Iraj Derakhshan \\ Formerly of Department of Neurology, \\ Case Western Reserve and Cincinnati \\ Universities, Ohio, USA
}

\begin{abstract}
The evidence that speech is the marker of hemisphere of action is overwhelming. Thus, contrary to the commonly accepted belief, the evidence indicates that both sides of the body are under the same command (major hemisphere) and that the nondominant side of the body is a callosum-width farther from the major hemisphere. Substantial controversy exists, however, as to the best method for determining the laterality of motor control in an individual case. According to the new understanding, ie, the one-way callosal traffic circuitry underpinning laterality of motor control, the larger excursions of effectors located opposite (contralateral to) the command center while performing bimanual simultaneous drawing tasks provides the best noninvasive and inexpensive approach for demonstrating the laterality of the major hemisphere of a person (who is able to perform such tasks). Here, it is documented pictorially that bimanual simultaneous drawing of geometrical designs or straight lines, as well as moving the arms simultaneously from side to side (or up and down) while noting the difference of speed of the two arms (represented by the distance between the two index fingers), both provide a reliable indication of the laterality of a person's major hemisphere. In all these maneuvers the nondominant side of the body (even the diaphragms) lags behind the dominant side by an interval equal to the interhemispheric transfer time (IHTT). This lagging behind of the nondominant side of the body in bimanual simultaneous movements is the footprint of directionality of callosal traffic underpinning the laterality of motor control evidenced by worsening of the delay of the nondominant side following callosotomy (uncoupling). Here, the historical precedence of a novel understanding in motor control together with its neurological implications in daily life as well as in laterality of seizure onset are briefly addressed, pointing out the deleterious effects of Sir Isaac Newton's influence in neurological research on interhemispheric connectivity by suggesting symmetrical representation of visual sense of space in the human brain.
\end{abstract}

Keywords: speech, brain, hemisphere, motor, control, callosotomy

\section{Introduction}

Much of what we know about motor abilities in humans is based on the performances of our hands as solo practitioners rather than on their cooperation when they act together at the same time. Various inventories are available for such solo performances, usually based on the use of different utensils employed in daily life (forks, knives, scissors, etc). We normally pay no attention to the asymmetry of facial movements as we speak (unless extreme in severity or when we are alerted about it in a particular case by someone more sensitive than ourselves) or to the sound of a drum struck by two sticks at the same time or to that of keyboard instruments requiring simultaneous performance by both hands.

Thus, few people realize the onomatopoeic nature of drummers" "flam," or know the neural underpinning of pianists" "melody lead of the right hand," as the latter (unsuccessfully) try to deliver two notes at the very same time (one for melody by the
Correspondence: Iraj Derakhshan 415 Morris st, Suite 40I, Charleston, WV 2530I, USA

Tel +l 3043434098

Email idneuro@hotmail.com 
right hand and the other for harmony by the left). Yet, such inescapable differences are visible to the naked eye if one is looking for them whenever we move our hands together to the sides or up and down. This is seen in Figure 1, chosen from two consecutive scenes of the movie, Hairspray (released in 2007). The same phenomenon, ie, wider excursion of the side opposite to the command center in a control subject is documented in Figure 2 which appeared in a study by Kennerley and colleagues (2002). The present article seeks to delineate the neural structures that dictate faster speed of one hand over the other as the hands move simultaneously towards their goal(s), with emphasis on "simultaneity" of movements, using insights from the one-way callosal traffic scheme documented elsewhere (Derakhshan 2005, 2006a, 2006c).

As it can be seen in Figure 1, the distance between the hands increases as the subject moves them simultaneously
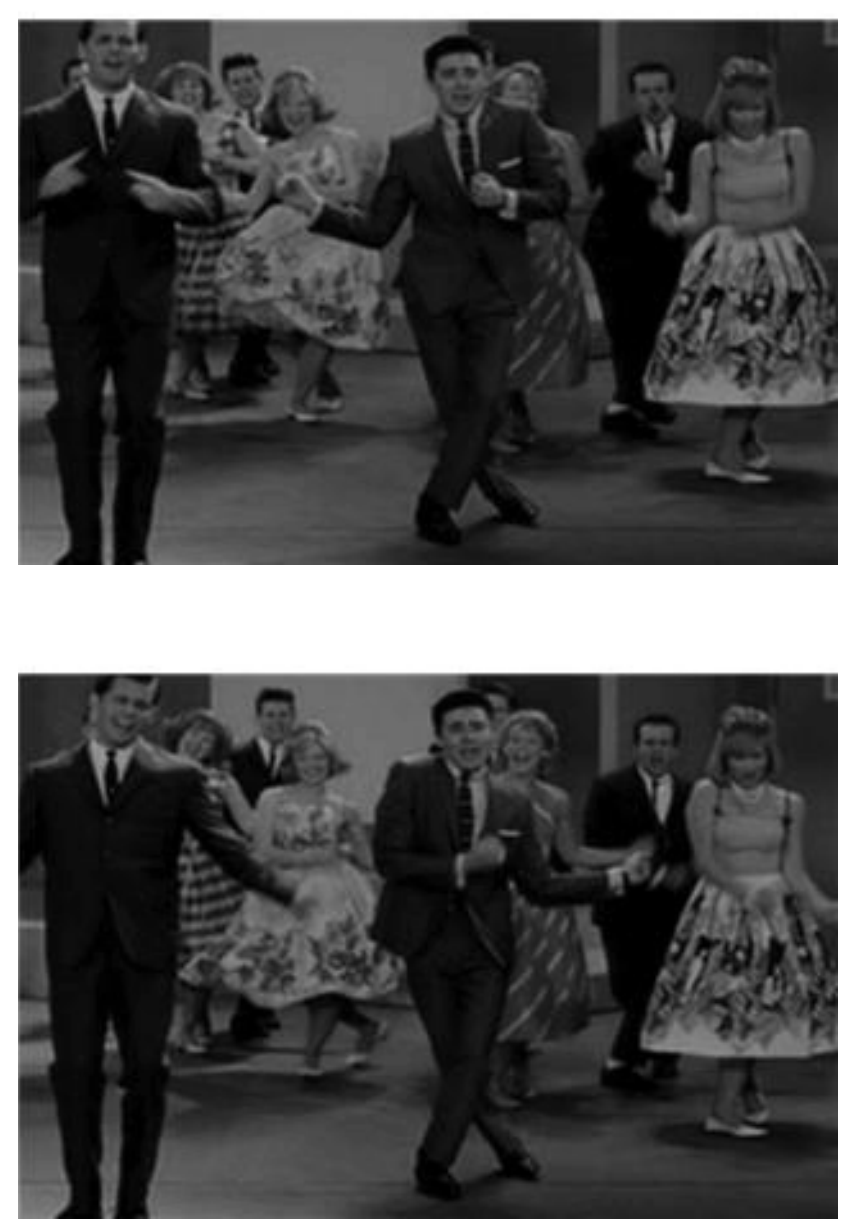

Figure I From two consecutive scenes in the movie, Hairspray. Note widening of the distance between two hands, as the artist (in the center) moves his hands simultaneously to the right. This is related to the invasion of the midline by the right arm seen in the scene below, indicating larger excursion by the dominant side. On the other hand respect for the midline by the nondominant hand is noted in the scene above. The larger excursion by the dominant hand means its faster speed as the hands move simultaneously from side to side. See text for further explanation. to the right and decreases as they move to the left. Clearly, the difference in distance between the two hands (in the two frames) is due to the crossing of midline (represented by the artist's tie) by the right hand as the hands move to the left (seen in the second frame). This situation will also occur if the hands were to move vertically as with the movements performed in piano playing (see below).

The reader may readily confirm this arrangement by moving his/her outstretched arms vertically at the same time (preferably with eyes closed), noting that one hand stays above the other as they open their eyes. Moreover, it is always the same hand that has moved faster regardless of where they had been before the race started (It is important that persons performing these movements do not engage in any other activity while doing the test, such as talking or humming. Doing so will detract more from the performance of the dominant side of the body due to the double-tasking involved in such events.)

With this understanding in mind, it is easy to realize that our ability to play string instruments, like violin or cello, is entirely dependent on this obligatory temporal precedence of the right hand (which holds the bow) to the fingering left hand (Baader et al 2005). How could we ever have made instrumental music otherwise, we may reasonably ask? How this phenomenon has come about and what is its relationship to our ordinary utensil-pegged handedness?

The answers may come from the recent evidence suggesting that that sensory and motor traffic across the corpus callosum and anterior commisure is one-way; ie, from the major to the minor hemisphere (for motor commands, crossing in the front of the high way mentioned), but from the minor to the major hemisphere (for sensory signals which had arisen from the nondominant side of the body, crossing in the back of the callosum on their way to the major hemisphere before they reach awareness by the subject [Derakhshan 2006a]).

In $80 \%$ of people the major hemisphere (command center) is located on the left side and in $20 \%$ on the right. Thus, the archaic formula of "right hemisphere controls the left side and left hemisphere controls the right," which is utterly incapable of explaining the asynchronies delineated above, should be abandoned in favor of the new understanding; ie, that all commands (including speech) are initiated in the same hemisphere, with the other hemisphere attending entirely to sensory and motor affairs of the nondominant side of the space, including moving the nondominant side of the body but always at the behest of the neighboring major hemisphere. Studies employing unimanual crank rotation tasks (using an Etch A Sketch toy) have elucidated the role of directionality 

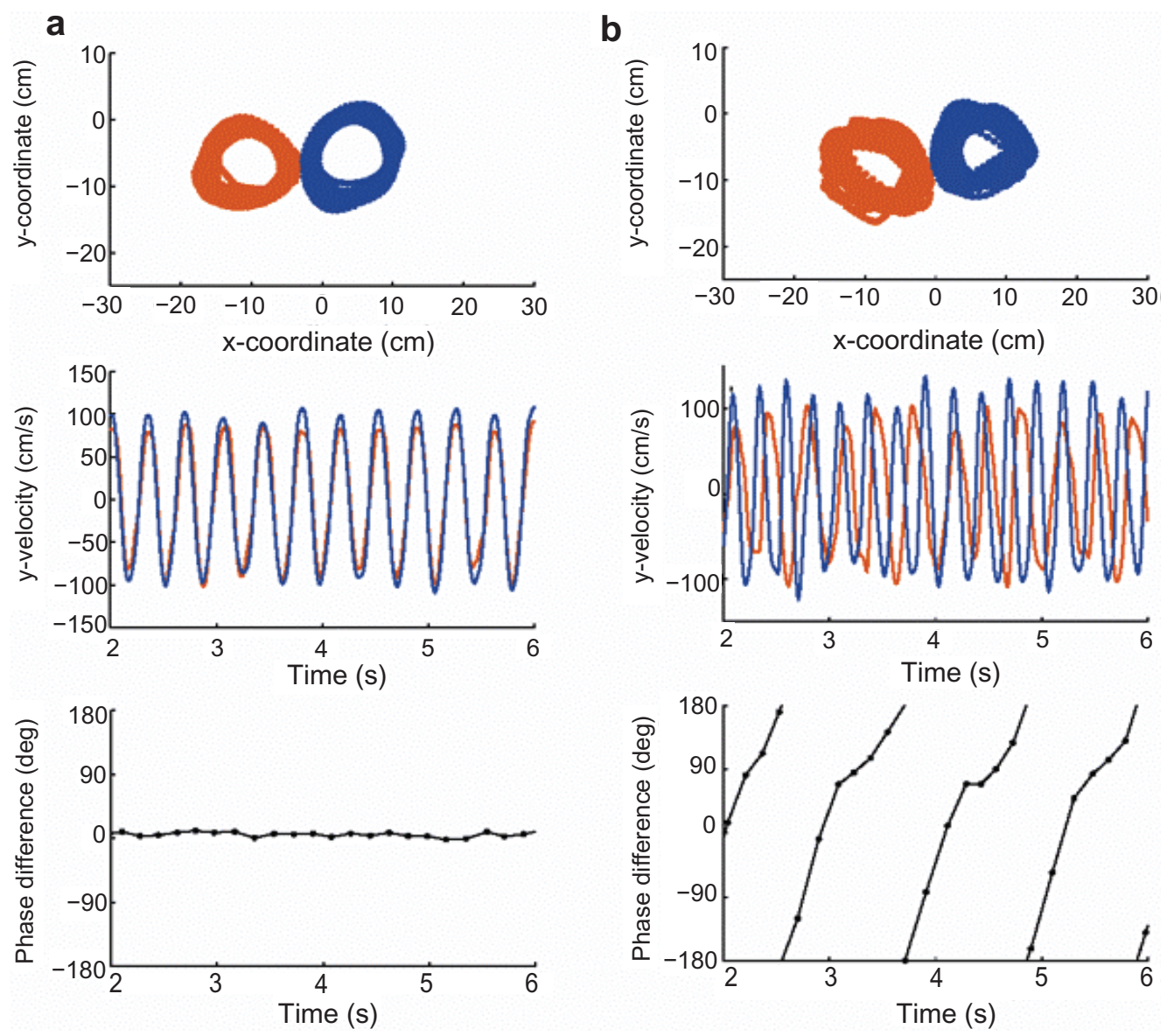

Figure 2 Temporal uncoupling during a single trial of symmetric, maximum rate circling in a patient. (a) Control subject. Note larger size of the circle drawn by the right hand (in blue). This finding was overlooked by the authors of the original study. (b) Callosotomy patient VP. Position (top) and velocity along the $y$-axis (middle) of all the cycles within a single trial for the left (red) and right (blue) hands. Bottom, relative phase relationship between the two hands. Point estimates of relative phase were calculated by determining the time of occurrence of every North/South point of the nondominant hand relative to two successive North/South points of the other hand. Negative values indicate a left-hand lead. The control participant shows synchronous movements with a stable phase relationship. For the patient, the right hand cycles at a higher frequency, causing a continuous drift in the phase relationship (that is, phase wrapping). Copyright @ 2002 . Reproduced with permission from Kennerley SW, Diedrichsen J, Hazeltine E, Semjen A, Ivry RB. 2002. Callosotomy patients exhibit temporal uncoupling during continuous bimanual movements. Nat Neurosci, 5:376-8I.

in callosal traffic in normals and in patients with callosal disease (eg, multiple sclerosis or those with callosal sectioning for intractable epilepsy) (Preilowski 1972; Kennerley et al 2002; Fagard and Corroyer 2003; Marion et al 2003; Bonzano et al 2008).

The same circuitry governs imagining moving the right or left hand, with those of the left associated with activation of both hemispheres, major hemisphere issues the command and minor hemisphere carries them out (Derakhshan 2006a). In an elaborate study by Maruff and colleagues (1999), the lagging of the left hand due to transcallosal transfer of command from the major to minor hemisphere was demonstrated in imagined movements.

It is to be noted that prior to the new insight afforded by one-way callosal traffic scheme the nondominant delay had usually been ascribed to the laziness of synaptic structures of the minor hemisphere governing the left side of the body (Tarkka and Hallett 1990; Swinnen et al 1996, 1998). More recently, however, the "need for revision" of abovementioned contralateral innervation model was sounded by Crone and colleagues (1998) after observing that moving the left side of the body alone was associated with cortical activation of both hemispheres. Similar data was debated fairly recently between the present author and Franz and colleagues (Derakhshan et al 2003). Furthermore, numerous time-resolved studies have shown that the excitatory signals controlling the minor hemisphere (right, in $\sim 80 \%$ of people), from the major, pass through the knee (genu) or the front third of the corpus callosum (Preilowski 1972; Nowicka and Fersten 2001; Kennerley et al 2002; Larson et al 2002; Sigman and Dehaene 2006; Spironelli et al 2006; Bonzano et al 2008).

From a historical perspective, one danger of venturing into an unfamiliar field by those whose authority in their 
own arena is unquestioned is the automatic transfer of that authority into the realm of which they may know almost nothing. Understandably, the consequences of this undeserved authority may be devastating. This situation occurred between the two disciplines of physics and biology in the twentieth century (the late physicist William Shockley's eugenics debacle may be cited as an example). However, the most devastating instance of this kind of authority-transfer occurred late in the seventeenth century with the forays into biology by a personality no less eminent than Sir Isaac Newton. It was Newton who suggested that fibers arising from nasal retina must of necessity cross at the chiasm (where the two optic nerves seemed to meet), allowing the representation of each hemispace in the opposite hemisphere (Horton 2006). It is easy however to show that this widely held belief is only a dogma since, except for an unreplicated article by Kupfer and colleagues (1967) to that effect, no evidence for such a decussation at the chiasm has ever existed. This much is apologetically admitted by the author of a chapter on the subject in an authoritative textbook of ophthalmology, stating: Indeed most of the classic and time honored anatomic schemata of the chiasm, upon which clinical interpretation of chiasmal visual field defects are made, are misleading and have little basis in fact (Slamovits 1998). Later, the same dogma was confounded by an older misunderstanding known as the Poffenberger paradigm (Derakhshan 2006a). Poffenberger grafted the Newtonian suggestion (ie, contralaterality of visual sense of space, with the two hemispaces meeting at the midline) with a centuries-old but partially plausible view concerning contralaterality of motor control (see for example, Sainburg 2002, p.253).

Thus, for too long we have come to believe that movements of the right side of the body in response to events occurring in the right visual field involves the left (verbal) hemisphere and those the left side to stimuli arising from the left side of the midline (hemispace) involve the right (spatial, nonverbal) hemisphere. However, as pointed out by some of the pioneers in the field of speeded response to lateralized stimuli, the "result of studies using reaction time to Stroop stimuli [the use of ink color and meaningful stimuli in tachistoscopic experiments with manual responses] to explore functional differences between the hemispheres has been disappointing" (Simon et al 1985).

Thus, Simon's and colleagues' study (1985), like other investigations reviewed by the author elsewhere (Derakhshan $2005,2006 a)$ confirmed that the asymmetry of manual reaction time is a function of the laterality of the responding hand (right or left), not the laterality of the visual field stimulated and that the right hand responded faster than the left regardless of the nature of the stimulus (verbal or nonverbal). The situation is the reverse in those who are truly wired as left-handers (Lutsep et al 1995). The falsity of examples to the contrary, recently cited by Goble (2007), will become clearer in the course of clarifying the distinction between behavioral (avowed) and neural handedness (brainedness, hemisphericity).

At the same time, questions concerning our knowledge of the external world, which is revealed to us at a glance, despite the above-claimed spatial dichotomy were left unanswered or hastily ascribed to a role played by the corpus callosum (Gazzaniga 2000; Derakhshan 2004). The evidence, including those derived from the removal of one-hemisphere (ie, minor hemisphere) and collosal section surgeries, points to the integrity of the central vision of those who under have gone such drastic surgical operations for varieties of medical reasons (Preilowski 1972; Derakhshan 2005, 2007). In the same vein, several cases of sudden loss of vision upon monohemispheric strokes are on record, sometimes associated with aphasia (Kraus et al 1990; Abutalebi et al 2007). With the absence of any role by the corpus callosum in macular vision thus established and insight derived from the role of directionality of callosal traffic in underpinning laterality of motor and sensory control (see above), I now recite a summary of the facts concerning the same and proceed to provide a description of quotidian manifestations of this circuitry so as to allow their verification by an interested observer using a simple paper and pencil test.

According to the new scheme, the side of the command center (ie, the major hemisphere) in all of us is revealed by the fact that moving the limb opposite to that hemisphere occurs at a faster speed than moving the side of the body lying directly beneath the command center (ie, the nondominant side). This is because moving the nondominant side must await the arrival of the command issued in the command center (where the decision maker resides). This communication occurs via the corpus callosum and anterior commisure. Thus, in all attempts to move the hands at the same time the nondominant hand remains stationary until the command issued in the command center for the purpose reaches that hand after the transfer of the command signal to the minor hemisphere. Throughout this interval, and despite the belief of the person performing the task to the contrary, the nondominant hand remains stationary. Direct demonstration of involvement of both hemispheres in moving the nondominant side of the body comes from varieties of time resolved electrical and blood circulation studies (Derakhshan 2003; 
Derakhshan et al 2003), including the well documented fact that, in right-handers, moving the left hand is associated with increased blood flow in both hemispheres while moving the right hand increases the blood flow of blood only to the left hemisphere regardless of the nature of visual paradigm used for the stimulation (verbal or nonverbal) (Kim et al 1993; Panerai et al 2005).

As a result, pianists, who play the notes written for melody (song) by the right hand and for harmony by the left, had long noted that the emergence of melody prior to harmony by $\sim 100 \mathrm{~ms}$ in such "simultaneous" performances. Musicologists had referred to the phenomenon as "the melody lead of the right hand in piano players," poetically ascribing the event to the artist's emotional state while playing the song (Vernon 1936). The same timing discrepancy may be displayed on a piece of paper by simultaneously drawing two straight lines in horizontal or vertical directions using both hands (preferably with pencils of different color and with eyes closed while making certain that nothing except the tips of the pencils touches the table on which drawing occurs [so as to prevent friction affecting the test]). It will be noted that the hand opposite to the command center will draw a longer and straighter line than does the nondominant counterpart (indicating better control of the dominant side and degradation of signals arising from the ipsilateral hemisphere as it crosses the callosum). Various permutation of this test may be performed, all declaring the validity of the newly described one-way callosal traffic circuitry. Figure 2 is an example of the size differential of simultaneous drawings of both hands in a normal subject, a finding that remained unrecognized (overlooked) by the authors of the study in which it originally appeared (Kennerley et al 2002).

It is of historical interest that the original description of this finding goes back to 1876 when a famous ophthalmic surgeon, Robert Brudenell Carter, searching for an improvement in the techniques of lens extraction, noted the following observation with perspicuity: "The left hand will lag behind the right hand and will move with less freedom so as to form a smaller curve and to form it more slowly." Around the same time in 1884, two neurophysiologists from Yale, Hall and Hartwell, noted that right-handed subjects bisected a graduated yard-stick to the left of midline as they moved their index fingers "at the same speed" along the edge of a yard-stick, having started from the opposing ends of the same (with their eyes closed). The opposite occurred in two left-handed subjects whose index fingers met to the right of the middle of the yard-stick under the same condition. Thus, once again the dominant hand moved at a faster speed, ie, made larger excursions, than the nondominant. The similarity of this observation to that of tactile and visual pseudoneglect can hardly be doubted (but the subject will not be discussed here further [Derakhshan and Oliveri 2005])

If the above formulation were accurate we expect that upon drawing a line of the same length, separately with each hand, the nondominant hand make larger excursions so as to achieve parity with the performance of its counterpart (ie, it needs more wiggle room). This expectation was fulfilled in several studies, two of which will be cited here: In a study by Mack and colleagues (1993) on 12 right-handed subjects employing a cinematographic method of measurement of hand displacement, the left elbow made larger excursions than the right by $50 \%$ in drawing letters and figures of the same size. More recently, in a study comprising three children groups spanning early to middle childhood (ie, 4,6 , and 8 year-olds) and an adult comparison group (all right-handed), Ringenbach and Amazeen (2005) measured the amplitude of movements of left and right hand as each group traced bimanually (at the same time) circles of the same size with their right and left hands to the sounds of a metronome. They too reported that the amplitudes of left hand movements were significantly higher than those of the right when tracing circles of the same size.

The last two experiments are conceptually related to another experiment conducted by Mcpherson and Renfrew (1953) on right- and left-handed subjects (40 in each group). Asked to judge the size of two disks of equal size (one in each hand held between the thumb and the index finger), $38 \%(25 / 40)$ of left- and 18\% (7/40) of right-handed group indicated that the disk in their dominant hand was smaller than that in the (nondominant) hand. According to one-way callosal traffic circuitry, since laterality of motor control resides exclusively in one hemisphere (Derakhshan 2005, 2006a), the above described judgment asymmetry must have been based on the fact that in tactile surveillance of the expanse of the object upon the command, the nondominant hand underwent wider excursions/dislocations in order to "size up" the same size disk being assessed by the dominant hand at the same time (Mack et al 1993; Ringenbach and Amazeen 2005). This discrepancy of the time taken from the command to the hands created the subjective impression that the disk on the nondominant side was larger than the one held by the dominant; with the slack taken up by the interhemispheric transfer time. According to the new scheme, those exceptions to the rule documented by McPherson and Renfrew (1953) where subjects in whom the behavioral handedness and neural wiring (hemisphericity) did not match. Incidentally, the discrepancy ratio 
in each of these handedness groups is similar to those obtained for the lead of the behaviorally dominant hand to the nondominant hand as documented in a recent reaction-time study by Shen and Franz (2005), using bimanual simultaneous button pressing tasks, and to the ratio seen for language laterality in left- and right-handed subjects indexed by aphasia following hemispheric stroke in a classical article by Goodglass and Quadfasel (1954). Similarly, binomial distribution of the hemisphere of onset of seizure as an index of laterality of motor control was $\sim 1 / 5$ in favor of the left hemisphere, as revealed in a recent electroencephalography and magnetoencephalography of 24 epileptics by Ossenblok and colleagues (2007) (18 left and 6 right hemisphere) and reviewed elsewhere by the author (Derakhshan 2006c).

Thus, recognition of the role of directionality in callosal traffic in underpinning the laterality of motor control has provided deeper understanding of the above-mentioned data and of myriad of other clinical and experimental observations long awaiting an explanation. As another example of the same phenomenon, and one related to a vital role played by the major hemisphere in maintaining life, one may cite the wider excursion of the right diaphragm which precede movements of the left regardless of whether one breaths quietly or deliberately (Carr et al 1994; Kiryu et al 2006).

\section{Discussion}

Although evidence for lateralization of the decision maker in one or the other hemisphere has been accumulating over the past many decades (Marzi et al 2001), the paradigms inherited from Newton and Poffenberger still dominate our thinking about hemispheric connectivity with such effectiveness that the essential meaning of those contrary findings thus far enumerated were never carried to their logical conclusion; ie, that the conscious element in our mind (the decision maker) resides in one hemisphere (the left, in vast majority of the population). This is in part due to the fact that the meaning of reaction time studies indicating quicker response by the dominant hand had not been fully understood; because of the outdated assumption that signals trafficking between the two hemispheres are symmetrical in directionality, redundantly sharing information between two homologous structures of the brain).

Several painstakingly conducted studies, however, had hinted the opposite to be the case. For example, in a land mark study of veterans who suffered penetrating brain injuries in World War II and the Korean campaigns, Semmes and colleagues (1960) of Harvard University documented that sensory deficits affecting the left hand may be associated with injury to either hemisphere, while those affecting the right hand was limited to injury to the left; indicating (in light of the new scheme, see above) a callosally mediated representation of the left hand in the left as well as the right hemisphere. This observation was later confirmed by Schwartz and colleagues (1979) in a cohort of 234 patients with unilateral brain damage and in a subsequent comprehensive review of time-resolved studies reviewed by the author (Derakhshan 2006a). A disconnection syndrome involving the left hand of a right-handed subject following a left hemispheric insult, resulting in aphasia and destruction of body of the callosum is also on record (a rarely reported event), again indicating duplicate representation of the left hand in the left hemisphere (Habib et al 1990). In the same vein, a study comprising 244 soldiers from the Vietnam era with penetrating skull wounds on one side which resulted in immediate loss of consciousness, loss of speech (aphasia) and epilepsy revealed a left to right hemispheric lateralization of the command center at $80 \%$ to $20 \%$, using speech as a marker of the major hemisphere (Mohr et al 1980).

In the present context, if we compare occurrences of the longer lines drawn by a group of normal people we will find that $\sim 80 \%$ will draw a longer line with their right hand and the remainder with their left hand. This was recently confirmed in a survey I did in a group musicians who played for the Symphony Orchestra in Charleston, West Virginia. Of the 50 musicians tested, 40 (80\%) drew longer lines with their right hand and $10(20 \%)$ with their left. As can be seen, this left to right hemispheric ratio of $80 / 20$ constitute a rough reflection of right to left handedness we observe at the society at large (Mohr et al 1980; Derakhshan 2006a), indicating a 15\%-20\% neurobehavioral disparity (mismatch) between avowed handedness and brainedness in the public. Neurologist have known of the disparity of some people's declared handedness with that for which they really were wired (using speech as a marker of the command center) since 1899 when the subject was first given the name of crossed aphasia by Byrom Bramwell, a British neurologist (Coppens et al 2002). Nevertheless the subject has been "puzzling" neurologists and neuroscientists alike ever since; as in the case of left-handed patient V. J. who acted like a right hander in her ability to pantomime object use and in superior temporal stability of her (neurally) dominant right hand after a callosotomy for seizure disorder (Ivry and Eliot 1999; Kennerley et al 2002; Frey et al 2005). Here, the fact that a group of investigators “puzzled” by V. J.'s self-declared (left) handedness (Ivry and Eliot 1999) continued to take the self-declared (right) handedness of their control subjects in another study at face value (Derakhshan et al 2004) despite 
the consistency of the left hand lead in reaction time study in three of those subjects points to presence of an unjustified bias in favor of the (now refuted) Newtonian paradigm of contralateral innervation. According to one-way callosal traffic circuitry, the three (of six) right-handed control participants studied by Diedrichsen and Ivry (2003), with consistent faster reaction times of their left hand, were indeed wired as right hemispheric participants, regardless of their declared handedness to the contrary.

In this context many authors had in the past spoken of "hidden" left handedness when trying to explain cases of crossed aphasia; an explanation which, in a round-about way, agrees with what is advocated by present writer based on time-resolved studies critiqued above (Diedrichsen et al 2003; Derakhshan et al 2004) as well as results of the bimanual simultaneous drawings mentioned earlier.

As mentioned earlier, the disparity of one's neural wiring (ie, which hemisphere "controls" the other) and adopted (avowed) handedness occurs in $\sim 20 \%$ of the population; numerically most of them among the right-handed. The role of vision in adopting one's favorite hand in early childhood is revealed in the facts that the incidence of left handedness among the congenitally blind is the same as that seen in general public while it increases several folds among family members of those who become left-handed (Lund 1933; Kutas et al 1975; Derakhshan 2003). In normal circumstances, the reasons for adopting a favorite hand other than that intended by nature (contralateral to the command center) is sentimental; eg, emulating a relative or friend who is left-handed or (less often), eschewing those who were left-handed for a perceived negative quality; as it was the case in a former left-hander who told me of her self-conversion to writing with the right hand at second grade so as not to appear like the few other left-handers in her school who were "disheveled."

Although it is customary to divide language into written and spoken, many people learn to communicate with sign language with its own grammar and vocabulary. The fact that these modalities of expression suffer the same consequences as a result of lesions affecting the same area of the major hemisphere (Meckler et al 1979) indicate that speech is best characterized as an act of symbolic significance and a marker of the action hemisphere. It is also relevant that all modalities of communication (including signing) are subject to the same neurobehavioral mismatch or uncertainty discussed above, ie, the occurrences of crossed aphasia and crossed nonaphasias in both handedness groups (Meckler et al 1979).

Thus, Meckler's signing patient who was left-handed, but displayed aphasia and right hemiplegia, was an instance of crossed aphasia in a left hander with his ostensibly dominant (left) side becoming of no use to him for signing purposes despite the fact that it was better off motor-wise than its completely paralyzed counterpart after the stroke. This case, however, typifies the essential difficulty (ie, nonhomogeneity of left-handers as a group) encountered by Sainburg (2002) for testing his dynamic hypothesis of handedness. He thus completely excluded left-handers from his studies, leaving the entire enterprise a questionable endeavor. As for the facts documented in right-handed subjects that Sainburg studied, ie, the faster speed of the right hand and wider excursion of the left arm for movements of the same amplitude as those by the right arm, they are similar to findings reported by others (Mack et al 1993; Ringenbach and Amazeen 2005) and in accord with predictions of the one-way callosal circuitry (see above).

On the other hand, Hund-Georgiadis's patient was a crossed nonaphasic dextral (ie, a neural left-hander with speech located in the right hemisphere) (Hund-Georgiadis et al 2002). This is a topic that has been reviewed extensively before under other names including that of "crossed right hemisphere syndrome" (Marchetti et al 2005). According to one-way callosal circuitry, those in this group of righthanded subjects are wired as left-handers and would not have acted according to the predictions of the dynamic dominance hypothesis espoused by Sainburg (2002). It appears to the writer that theories like those of Sainburg are designed to elevate the status of the right hemisphere to that of the left hemisphere among right-handed persons but in "visuospatial" domain, whereas the facts reviewed above indicate that such parity arises exclusively from the directionality of callosal traffic in individual subjects concerned regardless of their avowed handedness (see above).

To sum: In the laboratory the hand that is directly connected to the major hemisphere taps faster and with better regularity than its counterpart whose connection to the command center is indirect (via the corpus callosum and anterior commisure). It is also interfered with more than the other hand if a person engages in another task at the same time (like humming). The dominant hand will finish the job first while conducting a bimanual simultaneous activity (by an amount equal to IHTT). Clinically, injuries to the major hemisphere result in paralysis of both hands, with the effect on the nondominant side usually referred to as apraxia (rather than paralysis), whereas injury to the minor hemisphere or the callosum results in the paralysis of the nondominant side alone (Sakai et al 1998). Neglect and/or alienation of the left side of the body is the hallmark of lesions affecting 
the right hemisphere in a right-handed person, except in a neurally left-handed person where thing are in the opposite direction (Marchetti et al 2005). The person with injured right hemisphere denies the paralysis affecting his/her left side and even if he/she acknowledges the existence of paralysis he/she is likely to act with a great deal of detachment or to confabulate as to the reasons why the nondominant side does not move. Interestingly, moving the paralyzed side (to the extent possible) or irrigating the left ear canal (but not the right) improves the neglect commonly associated with nondominant paralysis (Derakhshan 2004). Since this phenomenon has never been reported without an extant anterior callosum it may be concluded that such improvements is the result of excitatory effect of the command signals arising from the left hemisphere, awakening a dormant (injured) right hemisphere into appreciating stimuli arising from the left (albeit temporarily and only while such stimulations last) (Derakhshan 2004). Anosognosia (denial of illness of the nondominant side) is caused by lack of callosal communication from the minor hemisphere's parietal cortex to its counterpart in the major hemisphere as it was documented by Green and Hamilton (1976), explaining the lack of awareness of the deficit on the nondominant side of the body.

Lesions of anterior corpus callosum or of the minor hemisphere are at times associated with development of the alien hand syndrome wherein unintended movement of the left hand creates the impression of a runaway extremity animated by a different mind.

Although dramatic in appearance, closer examination of the circumstances reveals that such movements are temporally related to those occurring in the right hand at or moments earlier (enabling movements), and do not indicate presence of a conscious element within the minor hemisphere (Scepkowski et al 2005). Occurrence of alien hand in the ostensibly dominant side of a patient points to the existence of neurobehavioral mismatch in the individual. Elsewhere, I have reviewed different aspects of such cases including those of Liepmann's Imperial counselor and that of renowned neuroanatomist Alf Brodal (Derakhshan 2006).

\section{Conclusion}

While the distinction between performances of the two hands has in the past been inventory driven (ie, based on the performance of each hand in right- and left-handed groups), it is shown here that far better insights might be gained by concentrating in temporal relationship between hands as they perform simultaneously while playing keyboards or string instruments or when drawing with both hands at the same time. Such activities in daily life clearly shows faster speed of the hand (dominant) that is directly connected to the command center than that of the other hand (nondominant) whose connection to the same center is indirect, ie, via the corpus callosum and anterior commissures. Evidence shows the following: a) comparing the size (amount of excursion) of such drawings is a valid method of determining a person's laterality of motor control as well as consciousness; b) when it comes to the laterality of motor control, it is the laterality of the command center that counts for certain, not a subject's avowed handedness. The latter is only a statistical reflection of the circuitry underpinning the laterality of motor and sensory control and does not correspond to the laterality of the command center in a sizable minority of humans.

\section{Disclosure}

The author reports no conflicts of interest in this work.

\section{Acknowledgments}

This article is dedicated to the memory of my mother and sister, Rebecca and Farkhondeh Derakhshan, whose kindnesses I will always remember.

\section{References}

Abutalebi J, Arcari C, Rocca MA, et al. 2007. Anton's syndrome following callosal disconnection. Behav Neurol, 18:183-6.

Baader AP, Kazennikov O, Wiesendanger M. 2005. Coordination of bowing and fingering in violin playing. Brain Res Cogn Brain Res, 23:436-43.

Bonzano L, Tacchino A, Roccatagliata L, et al. 2008. Callosal contributions to simultaneous bimanual finger movements. $J$ Neurosci, 19:3227-33.

Carter RB. 1876. A practical treatise on diseases of the eye. London: Macmillan, p. 145.

Carr LJ, Harrison LM, Stephens JA. 1994. Evidence for bilateral innervation of certain homologous motoneurone pools in man. J Physiol, 475:217-27.

Coppens P, Hungerford S, Yamaguchi S, et al. 2002. Crossed aphasia: an analysis of the symptoms, their frequency, and a comparison with lefthemisphere aphasia symptomatology. Brain Lang, 83:425-63.

Crone NE, Miglioretti DL, Gordon B, et al. 1998. Functional mapping of human sensorimotor cortex with electrocorticographic spectral analysis. I. Alpha and beta event- related desynchronization. Brain, 121:2271-99.

Derakhshan I, Franz EA, Rowse A. 2003. Handedness, neural versus behavioral: is there a measureable callosal difference. J Mot Behav, 35:409-14.

Derakhshan I. 2003. In defense of the sinistrals: anatomy of handedness and the safety of prenatal ultrasound. Ultrasound Obstet Gynecol, 21:209-12.

Derakhshan I. 2004. Handedness and macular vision: laterality of motor control underpins both. Neurol Res, 26:331-37.

Derakhshan I, Diedrichsen J, Hazeltine E, et al. 2004. Hugo Liepmann revisited, this time with numbers. J Neurophysiol, 91:2934-5.

Derakhshan I. 2005. Laterality of motor control revisited: directionality of callosal traffic and its rehabilitative implications. Top Stroke Rehabil, 12:76-82.

Derakhshan I, Oliveri M. 2005. Overestimation of numerical distances in the left side of space. Neurology, 64:1822-3. 
Derakhshan I. 2006a. Crossed-uncrossed difference (CUD) in a new light: anatomy of the negative CUD in Poffenberger's paradigm. Acta Neurol Scand, 113:203-8.

Derakhshan I. 2006b. Laterality of the alien hand: Directionality of callosal traffic underpins neural handedness. Accessed on July 20, 2008. URL: http://www.ucmss.com/books/LFS/CSREA2006/MLM3165.pdf

Derakhshan I. 2006c. Laterality of seizure onset and the simple reaction time: revamping the Poffenberger's paradigm for seizure surgery. Neurol Res, 28:777-84.

Derakhshan I. 2007. Nature's shell game revealed: evidence for non-Newtonian laterality of macular vision (Ocular integration in the human visual cortex). Can J Ophthalmol, 42:485-6.

Diedrichsen J, Hazeltine E, Nurss WK, et al. 2003. The role of the corpus callosum in the coupling of bimanual isometric force pulses. J Neurophysiol, 90:2409-18.

Fagard J, Corroyer D. 2003. Using a continuous index of laterality to determine how laterality is related to interhemispheric transfer and bimanual coordination in children. Dev Psychobiol, 43:44-56.

Frey SH, Funnell MG, Gerry VE, et al. 2005. A dissociation between the representation of tool-use skills and hand dominance: insights from left- and right-handed callosotomy patients. $J$ Cogn Neurosci, 17:262-72.

Gazzaniga MS. 2000. Cerebral specialization and interhemispheric communication: does the corpus callosum enable the human condition? Brain, 123:1293-326.

Goble D. 2007. Validity of using reaction time as a basis for determining motor laterality. J Neurophysiol, 97:1868.

Green JB, Hamilton WJ. 1976. Anosognosia for hemiplegia: somatosensory evoked potential studies. Neurology, 26:1141-4.

Habib M, Ceccaldi M, Poncet M. 1990. [Callosal disconnection syndrome caused by left hemisphere infarction.] Rev Neurol (Paris), 146:19-24.

Hall GS, Hartwell EM. 1884. Bilateral asymmetry of function. Mind, 9:93-109.

Goodglass H, Quadfasel FA. 1954. Language laterality in left-handed aphasics. Brain, 77:521-48.

Horton JC. 2006. Ocular integration in the human visual cortex. Can J Ophthalmol, 41:584-93.

Hund-Georgiadis M, Zysset S, Weih K, et al. 2002. Crossed nonaphasia in a dextral with left hemispheric lesions: a functional magnetic resonance imaging study of mirrored brain organization. Stroke, 32:2703-7.

Ivry RB, Eliot H. 1999. Subcortical locus of temporal coupling in the bimanual movements of a callosotomy patient. Hum Mov Sci, 18:345-75.

Kennerley SW, Diedrichsen J, Hazeltine E, et al. 2002. Callosotomy patients exhibit temporal uncoupling during continuous bimanual movements. Nat Neurosci, 5:376-81.

Kim SG, Ashe J, Hendrich K, et al. 1993. Functional magnetic resonance imaging of motor cortex: hemispheric asymmetry and handedness. Science, 261:615-17.

Kiryu S, Loring SH, Mori Y, et al. 2006. Quantitative analysis of the velocity and synchronicity of diaphragmatic motion: dynamic MRI in different postures. Magn Reson Imaging, 24:1325-32.

Kraus E, Meyer E, Zonis S. 1986. Sudden loss of vision in a monocular patient, caused by visual cortex infarction. Ann Ophthalmol, 18:114-15.

Kutas M, McCarthy G, Donchin E. 1975. Differences between sinistrals' and dextrals' ability to infer a whole from its parts: a failure to replicate Neuropsychologia, 13:455-64.

Kupfer C, Chumbley L, Downer JC. 1967. Quantitative histology of optic nerve, optic tract and lateral geniculate nucleus of man. J Anat, 101:393-401.

Larson EB, Burnison DS, Brown WS. 2002. Callosal function in multiple sclerosis: bimanual motor coordination. Cortex, 38:201-14.

Lund FH. 1933. Psychology: An empirical study of behavior. New York: The Ronald Company, pp. 189-92.

Lutsep HL, Wessinger CM, Gazzaniga MS. 1995. Cerebral and callosal organisation in a right hemisphere dominant "split brain" patient. J Neurol Neurosurg Psychiatry, 59:50-4.

McPherson A, Renfrew S. 1953. Quarterly of perception of size between the right and left hands in normal subjects. Q J Exp Psychol, 5:66-74.
Marion SD, Kilian SC, Naramor TL, et al. 2003. Normal development of bimanual coordination: visuomotor and interhemispheric contributions. Dev Neuropsychol, 23:399-421.

Mack L, Gonzalez Rothi LJ, Heilman KM. 1993. Hemispheric specialization for handwriting in right handers. Brain Cogn, 21:80-6.

Maruff P, Wilson PH, De Fazio J, et al. 1999. Asymmetries between dominant and non-dominant hands in real and imagined motor task performance. Neuropsychologia, 37:379-84.

Marchetti C, Carey D, Della Sala S. 2005. Crossed right hemisphere syndrome following left thalamic stroke. J Neurol, 252:403-11.

Marzi CA, Girelli M, Natale E, et al. 2001. What exactly is extinguished in unilateral visual extinction? Neurophysiological evidence. Neuropsychologia, 39:1354-66.

Meckler RJ, Mack JL, Bennett R. 1979. Sign language aphasia in a nondeaf-mute. Neurology, 29:1037-40.

Mohr JP, Weiss GH, Caveness WF, et al. 1980. Language and motor disorders after penetrating head injury in Viet Nam. Neurology, 30:1273-9.

Nowicka A, Fersten E. 2001. Sex-related differences in interhemispheric transmission time in the human brain. Neuroreport, 12:4171-5.

Ossenblok P, de Munck JC, Colon A, et al. 2007. Magnetoencephalography is more successful for screening and localizing frontal lobe epilepsy than electroencephalography. Epilepsia, 48:2139-49.

Panerai RB, Moody M, Eames PJ, et al. 2005. Dynamic cerebral autoregulation during brain activation paradigms. Am J Physiol Heart Circ Physiol, 289:H1202-8.

Preilowski BF. 1972. Possible contribution of the anterior forebrain commissures to bilateral motor coordination. Neuropsychologia, 10:267-77.

Ringenbach SD, Amazeen PG. 2005. How do children control rate, amplitude and coordination stability during bimanual circle drawing? Ecol Psychol, 17:1-18.

Sainburg RL. 2002. Evidence for a dynamic-dominance hypothesis of handedness. Exp Brain Res, 142:241-58.

Sakai K, Kojima E, Suzuki M, et al. 1998. Primary motor cortex isolation: complete paralysis with preserved primary motor cortex. J Neurol Sci, 155:115-19.

Scepkowski LA, Cronin-Golomb A. 2003. The alien hand: cases, categorizations, and anatomical correlates. Behav Cogn Neurosci Rev, 2:261-77.

Schwartz AS, Marchok PL, Kreinick CJ, et al. 1979. The asymmetric lateralization of tactile extinction in patients with unilateral cerebral dysfunction. Brain, 102:669-84.

Semmes J, Weinstein S, Ghent L, et al. 1960. Somatosensory changes after penetrating brain wounds in man. Boston: Harvard University Press, chapter 4.

Shen YC, Franz EA. 2005. Hemispheric competition in left-handers on bimanual reaction time tasks. J Mot Behav, 37:3-9.

Sigman M, Dehaene S. 2006. Dynamics of the central bottleneck: dual-task and task uncertainty. PLoS Biol, 4:e220.

Simon JR, Paullin C, Overmyer SP, et al. 1985. Reaction time to word meaning and ink color of laterally-presented stroop stimuli: effects of handedness and sex. Int J Neurosci, 28:21-33.

Slamovits TL. 1998. Anatomy and physiology of the optic chiasm. In: Miller NR, Newman NJ (Eds). Walsh and Hoyt's Clinical Neuro-Ophthalmology. 5th Edition, Vol. 1. Baltimore, MD: Williams and Wilkins, p. 91.

Spironelli C, Tagliabue M, Angrilli A. 2006. Asymmetrical hemispheric EEG activation evoked by stimulus position during the Simon task. Neurosci Lett, 399:215-19.

Swinnen SP, Jardin K, Meulenbroek R. 1996. Between-limb asynchronies during bimanual coordination: effects of manual dominance and attentional cueing. Neuropsychologia, 34:1203-13.

Swinnen SP, Jardin K, Verschueren S, et al. 1998. Exploring interlimb constraints during bimanual graphic performance: effects of muscle grouping and direction. Behav Brain Res, 90:79-87.

Tarkka IM, Hallett M. 1990. Cortical topography of premotor and motor potentials preceding self-paced, voluntary movement of dominant and non-dominant hands. Electroencephalogr Clin Neurophysiol, 75:36-43.

Vernon LN. 1936. Synchronization of chords in artistic piano music. In: Seashore CE (Ed). Studies in Psychology of Music. Vol. 3. Iowa City, IA: University Press, pp. 306-45. 
